\title{
Féeries
}

Études sur le conte merveilleux, XVII ${ }^{\mathrm{e}} \mathrm{XIX}{ }^{\mathrm{e}}$ siècle

$8 \mid 2011$

Le merveilleux français à travers les siècles, les langues, les continents

\section{Un remake de La Barbe bleue, ou l'au-revoir à Perrault}

Remaking Bluebeard, or Good-bye to Perrault

Jack Zipes

Traducteur : Cécile Boulic

\section{OpenEdition}

Journals

Édition électronique

URL : http://journals.openedition.org/feeries/779

DOI : 10.4000/feeries. 779

ISSN : 1957-7753

Éditeur

UGA Éditions/Université Grenoble Alpes

Édition imprimée

Date de publication : 15 octobre 2011

Pagination : $71-90$

ISBN : 978-2-84310-211-0

ISSN : $1766-2842$

Référence électronique

Jack Zipes, «Un remake de La Barbe bleue, ou l'au-revoir à Perrault», Féeries [En ligne], 8 | 2011, mis en ligne le 15 avril 2013, consulté le 07 septembre 2020. URL : http://journals.openedition.org/feeries/ 779 ; DOI : https://doi.org/10.4000/feeries.779 
Jack Zipes

The University of Minnesota (USA)

\title{
UN REMAKE DE LA BARBE BLEUE, OU L'AU-REVOIR À PERRAULT
}

\begin{abstract}
"Quand j'étais enfant, c'était mon conte préféré, mais je m'étonnais que cette histoire soit racontée aux petites filles, parce que c'est un conte dans lequel les femmes se font tuer : Barbe bleue est un vrai tueur en série. Dans les contes le protagoniste est souvent un ogre - comme dans Le Petit Chaperon rouge — qui éprouve le besoin de dévorer ses victimes pour se nourrir. Mais dans le cas de Barbe bleue, il s'agit d'un être humain qui se marie avec ses victimes, dont cette jeune femme. Mais dans un certain sens, il est aussi naif que MarieCatherine.

Si vous visionnez mes films, vous verrez que je suis quelque peu obsédée par la relation entre les victimes et leur bourreau, comme si la relation était rationnelle au sens physique du terme, comme si c'était une relation entre deux forces distinctes qui se mesurent l'une à l'autre. Et c'est pourquoi j'ai toujours souhaité réaliser un film sur Barbe bleue ${ }^{\mathrm{I}}$.»
\end{abstract}

Catherine Breillat.

\begin{abstract}
7 Harles PeRRaUlT Ne SAVAit Pas, lorsqu'il a créé ce conte sur le tueur en série Barbe bleue, qu'au xxI siècle, son méchant serait devenu une figure mémétique dans la plupart des sociétés occidentales. De plus, s'il vivait aujourd'hui, Perrault serait surpris d'apprendre que son cruel assassin a été l'objet de centaines si ce n'est de milliers d'interprétations, de manipulations et de transformations. En fait, il serait certainement ébahi pour ne pas dire abasourdi par ce qu'il a inventé.
\end{abstract}

I. P. Jahn, «Bluebeard : Interview with Catherine Breillat», Electric Sheep (i6 juillet 2010) : http://www.electricsheepmagazine.co.uk/features/2010/o716/bluebeard 
Peu après sa parution en 1697 , le conte de Perrault fut adapté de façon inhabituelle dans des chapbooks et des représentations théâtrales, et traduit en différentes langues européennes. Il a aussi été remanié durant les quatre derniers siècles à travers d'autres formes artistiques et culturelles : opéra, théâtre, affiches, cartes postales, poésie, radio, roman, livres pour enfants, bandes dessinées, cinéma, photographie, peinture, télévision et représentations numériques sur Internet. De plus, rien que ces dix dernières années, quatre longs travaux universitaires ont été menés: The Tale of Bluebeard in German Literature: From the Eighteenth Century to the Present de Mererid Puew Davies, paru en 2001, Secrets beyond the Door: The Story of Bluebeard and his Wives de Maria Tatar en 2004, Bluebeard: A Reader's Guide to the English Tradition de Casie Hermansson en 2009 et Tales of Bluebeard and his Wives from Late Antiquity to Postmodern Times de Shuli Barzilai en 2009. Tous révèlent que les histoires de tueurs en série dans les cultures occidentales et du Moyen-Orient sont tout à fait courantes depuis l'Antiquité jusqu'à aujourd'hui, et ils montrent comment $\mathrm{La}$ Barbe bleue de Perrault est devenue l'œuvre maîtresse. De manière intéressante, et bien que tué par Perrault, Barbe bleue n'est pas mort. Plutôt, il a été ressuscité des milliers de fois et a pris de nombreuses formes différentes, tandis que la paternité de Perrault sur La Barbe bleue a perdu de l'importance et est généralement négligée. Malgré la grande attention que les universitaires prêtent à Perrault, son nom a été plus ou moins effacé de la mémoire populaire, alors que le nom de Barbe bleue demeure.

Il y a quelque chose d'étrange dans les processus d'adaptation et de diffusion des histoires orales et des contes littéraires, car il semble que dès que le récit devient assez pertinent et digne d'intérêt dans certaines sociétés, il est récupéré de façon mémétique par une grande partie de la population qui néglige l'auteur pour s'approprier le conte ou pour rendre honneur au conte lui-même. Michael Drout précise qu' «un mème est le plus petit élément de réplication culturelle; tout élément qui est communiqué quand une personne en imite, consciemment ou non, une autre ${ }^{2}$ » et il démontre comment les mèmes créent les traditions orales et littéraires à travers les processus d'imitation et de répétition.

Une proto-tradition [des mèmes] pourrait facilement être créée par une civilisation à partir de ses épreuves et de ses erreurs, et être très largement répandue grâce aux penchants humains qui consistent à répéter des actions qui semblent donner de bons

2. M. Drout, "A Meme-Based Approach to Oral Traditional Theory», p. 269. Pour une étude approfondie sur les mèmes et les contes, je vous renvoie à mon ouvrage Why Fairy Tales Stick: The Evolution and Relevance of a Genre. Sur les mèmes, voir K. Distin, The Selfish Meme. 
résultats, à imiter leurs semblables couronnés de succès, et à enseigner aux générations suivantes les informations dignes d'intérêt ${ }^{3}$.

Bien que nous ne sachions pas si Perrault avait connaissance d'un conte oral en particulier qui avait pour titre La Barbe bleue — en réalité les conteurs donnaient rarement des titres à leurs histoires du temps de Perrault —, il avait certainement entendu ou lu des contes sur de célèbres tueurs en série, comme Gilles de Rais. Il connaissait très probablement l'histoire biblique d'Adam et Ėve, et la façon dont la curiosité d'Ève les expulsa du jardin d'Eden. Il connaissait Pandore et la façon dont sa curiosité l'amena à ouvrir la boîte à malheurs tant redoutée. Il devait même connaître Minos le roi de Crète, qui était la cause de fins tragiques pour les femmes avec qui il avait des relations sexuelles ${ }^{4}$. Il est impossible de dire combien de contes il avait en tête, des contes qui faisaient partie de son éducation, de sa culture et de ses habitudes. Mais il en était sans doute imprégné d'une certaine manière, car ils jouèrent un rôle dans la tradition culturelle française. Une fois qu'il a commencé à écrire son propre récit au sujet d'un meurtrier sans pitié avec une étrange barbe - qui pouvait être une caricature de son ennemi juré Nicolas Boileau -, les gens aimèrent le lire ou l'écouter et ils mémorisèrent des fragments de ce conte. À leur tour, certains publièrent leurs interprétations sur le conte de Perrault dans différents textes et contextes. D'autres se contentèrent de le raconter à leur manière, de le retenir et de s'y référer de temps en temps. De cette façon, La Barbe bleue de Perrault entra également dans la tradition orale, ou vint compléter des contes déjà existants qui traitaient eux aussi de tueurs en série. Alors que le nom de Perrault a généralement été associé au texte dans les éditions imprimées, ce n’est pas le cas dans de nombreuses traductions, livres pour enfants et dans d'autres moyens d'expression, notamment les représentations filmées. Presque tous les Barbe bleue mis sur écran que j'ai analysés dans mon ouvrage intitulé The Enchanted Screen, en partant du Barbe bleue comique et bizarre de George Méliès (1904) et jusqu'au Barbe bleue scintillant de Catherine Breillat (2009), ne se réferent qu'à peine, pour ne pas dire pas du tout, à Perrault. L'auteur est remplacé par le fameux méchant de son œuvre, comme l'a démontré Hans Christian Andersen à travers son célèbre conte L'Ombre dans lequel son protagoniste, un poète, a été insidieusement occulté par sa propre création. Barbe bleue éclipse Perrault.

3. Ibid., p. 27I.

4. Voir "Butchering Girls", dans G. Anderson, Fairytale in the Ancient World, Londres, Routledge, 2000, p. 97-I00. 
L'éclipse, la transformation et la ré-interprétation sont des composantes fonctionnelles majeures dans un processus de mémétique. Elles permettent à un conte donné de devenir populaire et classique dans la civilisation occidentale - et dans le cas de La Barbe bleue, particulièrement dans la culture française. À cet égard, la récupération de La Barbe bleue de Perrault par Breillat est extrêmement significative : elle révèle l'évolution historique du conte depuis la tradition orale, en passant par les éditions papier, et jusqu'à la représentation numérique et cinématographique d'aujourd'hui. La disparition de Perrault — et finalement le nom de Breillat disparaitra lui aussi - montre à quel point les contes et les histoires dépendent beaucoup de la résonance dont ils bénéficient dans de grands pans de la société et de leurs développements culturels. Les contes sur Barbe bleue et ses variantes dans d'autres domaines de la production culturelle, comme le cinéma, agissent comme des mèmes avec des stimuli extraordinaires et font partie d'un procédé discursif du remake dans le genre plus vaste du conte. Et je souhaite étudier La Barbe bleue de Breillat en tant que ré-interprétation filmée pour comprendre comment les contes oraux et littéraires sont entrés en interaction avec de nouveaux moyens d'expression, dans une longue tradition historique, afin de créer un langage propre au conte qui prenne en charge les changements de mœurs, de comportements et de valeurs.

\section{Comprendre le remake}

Tout d'abord, un mot concernant la définition du "remake», qui a généralement été utilisé en tant que terme technique dans le domaine de la recherche cinématographique pour désigner et classer un film créé à partir d'un autre film avec l'intention de l'expliquer, de l'améliorer, ou de le critiquer d'une façon inédite. Il peut également y avoir des remakes de remakes, ou des remakes fragmentaires avec des références intertextuelles. Ce qu'il est important de dire ici, c'est que la notion de ré-interprétation n'est pas réservée au domaine des études cinématographiques et de la production filmique, car presque chaque histoire racontée ou écrite est une sorte de remake. Un texte pur, original, ou urtext, n'existe pas, même quand une histoire retrace simplement une vie qui paraît unique ou assez significative pour être communiquée sous une forme narrative. On ne raconte ni ne répète la même histoire sans faire d'une certaine façon une nouvelle version de sa vie ou d'un récit; on n'écrit pas non plus des histoires sans reformuler d'autres histoires, sans se référer à d'autres, sans revivre des 
expériences et des rêves, et sans reconsidérer un genre. Charles Perrault, par exemple, était un écrivain très érudit, et quand il inventait ou écrivait ses contes, en les reprenant au besoin deux fois ou plus, il faisait référence à la littérature latine classique, aux mythes, à la Bible, aux contes des $\mathrm{XVI}^{\mathrm{e}}$ et $\mathrm{XVII}^{\mathrm{e}}$ siècles, à la poésie française contemporaine, aux fables, aux novellas, aux contes oraux qu'il avait entendus étant enfant et qu'il avait re-racontés à ses propres enfants, et à des contes écrits par de nombreuses femmes françaises talentueuses, comme Mademoiselle Lhéritier, sa nièce, et Madame d'Aulnoy, une connaissance, qui souvent les jouaient sur scène ou en parlaient dans les salons. Il faisait référence aussi aux différends qui l'opposèrent à Boileau et à d'autres intellectuels français de son époque à propos de littérature et de politique. Tout cela forme son habitus narratif, un concept qu'Arthur Frank, influencé par Pierre Bourdieu, a défini ainsi :

[une]... disposition à entendre certaines histoires comme étant celles que l'on doit écouter, que l'on doit répéter à des occasions précises, et que l'on doit suivre à la lettre [...]. L'habitus narratif implique un répertoires d'histoires qu'au moins une personne peut reconnaître et qu'un groupe partage. Ces histoires sont révélées par contraste avec un fonds invisible contenant toutes les histoires ignorées par cette personne et les histoires qui ne sont diffusées au sein d'aucun groupe en particulier ${ }^{6}$.

En effet, Perrault a digéré les biens culturels circulant autour de lui, et son intention sous-jacente était de re-créer et de célébrer la gloire d'une littérature française "moderne» qui incluait un genre prétendument mineur : le conte. Madame d'Aulnoy appela ce genre le conte de fée ${ }^{7}$, dont la source se trouvait dans le conte oral merveilleux et la littérature médiévale. Tous les auteurs talentueux du conte des années 1690 et du début du XVIII ${ }^{\mathrm{e}}$ siècle s'adonnaient au "travail» du remake, et leur "assiduité ${ }^{8} \mathrm{a}$ eu, au cinéma, de profondes répercussions sur la manière de réaliser des remakes. En retour, ces remakes cinématographiques créés aux $\mathrm{Xx}^{\mathrm{e}}$ et $\mathrm{XXI}^{\mathrm{e}}$ siècles illustrent à quel point le genre du conte de fée a évolué depuis le XVII ${ }^{\mathrm{e}}$ siècle et a prospéré de façon surprenante; ils se font le reflet des procédés culturels qui engendrèrent ce genre.

De nombreux essais et ouvrages ont été écrits sur l'adaptation filmée des romans et des histoires, et tous nous éclairent d'une façon ou d'une autre.

5. N.D.T. : souligné par l'auteur.

6. A. Frank, Letting Stories Breathe: A Socio-Narratology, Chicago, University of Chicago Press, 2010, p. 53.

7. N.D.T. : souligné par l'auteur.

8. N.D.T. : guillemets de l'auteur. 
Pour moi, le plus utile et le plus complet est l'ouvrage de Constantine Verevis, Film Remakes, publié en 2006. Dans sa préface, il déclare :

Tirant partie des récentes théories sur le genre et l'intertextualité, Film Remakes situe le remake dans une position élastique et complexe, rendue possible et à la fois limitée par les rôles interdépendants et les habitudes de cette industrie, de la critique et du public ${ }^{9}$.

Qui dit industrie du film dit problèmes de production, comme l'exploitation commerciale et les droits d'auteurs; qui dit critique dit étude des genres, des intrigues et des structures; qui dit public dit questions de réception et d'institutions.

Au terme de cette discussion, le remake cinématographique apparaît comme un cas particulier de répétition, relevant à la fois des domaines cinématographique et discursif et structuré par des pratiques historiques spécifiques : les règles du copyright, les droits d'auteur, la création d'une référence canonique, l'intelligence de ce moyen d'expression et les critiques de films ${ }^{\mathrm{IO}}$.

Bien que C. Verevis mette essentiellement l'accent sur les remakes filmés, il pose les bases d'un travail préparatoire pour une étude des procédés culturels qui mènent à la formation de nombreux autres genres littéraires et artistiques. En ce qui concerne le conte, il montre les faiblesses des approches textuelle et structurelle — tributaires d'une lecture au plus près des textes et intertextes - qui ignorent les facteurs extra-textuels contribuant au développement du genre et de ses remakes.

Comme tout concept critique (y compris le genre), le remake - citation, allusion, adaptation - est créé et corroboré par l'usage répété d'une terminologie qui lui est propre. On imagine que les références directes aux intertextes entre le remake et l'original sont très limitées, et qu'elles sont organisées autour de références extra-textuelles elles-mêmes contenues dans des formes de discours spécifique ancrées dans l'Histoire — notamment les critiques de films, mais aussi les règles du copyright, les droits d'auteur, la création d'une référence canonique et l'intelligence du film. Mais cette façon de penser a des conséquences sur les descriptions purement textuelles du remake, en particulier celles qui cherchent à figer cette catégorie dans une différenciation étroite entre l'histoire originale et sa nouvelle adaptation discursive. Parallèlement à la tendance discutable qui consiste à penser que l'essence immuable d'un scénario peut en quelque sorte être extraite des qualités innovantes de son expression, la différenciation entre l'intrigue et la manière de l'exprimer est de toute évidence déjouée par ces remakes qui non seulement reprennent l'invention narrative d'une ouvre

9. C. Verevis, Film Remakes, p. vii.

Io. Id. 
originale mais cherchent, en l'occurrence, à recréer la tonalité expressive d'un film déjà existant ${ }^{\mathrm{II}}$.

Concernant le conte, la théorie de C. Verevis sur le remake abat les frontières entre l'oralité, la littérarité, les arts visuels et les technologies de représentation pour montrer que chaque genre culturel est souple et subit, à des moments et des endroits particuliers, des changements causés par l'interaction de l'intertextualité et de l'extratextualité. L'interprétation ou les interprétations d'un conte ne peuvent pas provenir uniquement du regard porté sur le contenu textuel et intertextuel, mais doivent aussi intégrer une analyse des éléments de production et de réception sur les plans personnel, social et culturel. Le texte n'est qu'une série de lettres imprimées sur du papier qui ne demande qu’à être déchiffré; il n'a aucun sens tant qu'il ne se trouve pas entre les mains de lecteurs implicites - de lecteurs qui ne sont ni complices dans sa production ni dans son obligatoire réception. Le genre de ce qui a fini par être connu sous le nom de conte de fée est un procédé historique, mémétique et discursif, constitué par l'oralité et les moyens d'expression technologiques comme l'imprimerie, la radio, le cinéma et Internet. Pour comprendre le genre du conte et la relation du texte de Perrault avec le film de Breillat, nous pourrions tout d'abord tirer profit d'une étude de la généricité du conte.

\section{Généricité}

Dans leur récent ouvrage, Textualité et intertextualité des contes: Perrault, Apulée, La Fontaine, Lhéritier, Ute Heidmann et Jean-Michel Adam ont développé de façon significative leur utile notion de généricitét2 pour tenter d'élargir la notion de genre, et pour transcender les limites d'une approche textuelle et intertextuelle aux contes. Ils proposent de remplacer la catégorie de "genre" par la notion plus discursive de "généricité", un terme dont j'ai déjà parlé dans Why Fairy Tales Stick. Dans leur élaboration de "généricité» la plus récente, ils déclarent:

Ce concept désigne le processus d'inscription d'un énoncé dans une communauté discursive donnée. Il permet «d'éviter l'écueil essentialiste» qui rend toute comparaison interlinguistique et interculturelle impossible. La généricité se place en effet du côté "de la fluctuation, de l'instabilité, de la constante recatégorisation "; elle est inséparable de la variation du système de genres d'une époque ou d'un groupe social.

II. Ibid., p. 29.

I2. N.D.T. : en français et souligné dans le texte. 
Dans cette optique, il est moins question de classer un texte dans un genre comme «le conte» que de mettre en évidence les “tensions génériques" qui le traversent ${ }^{13}$.

U. Heidmann et J.-M. Adam cherchent à se démarquer des formalistes qui effectuent essentiellement des lectures approfondies des textes sans prendre en compte leur contexte, et ils critiquent constamment les folkloristes qui s'appuient sur l'étude de contes-types et "universalisent» les interprétations des contes sans prendre en compte leur contexte socioculturel propre ni leurs significations intertextuelles. Ils veulent comprendre comment les éléments d'un texte entrent en écho avec d'autres textes, parfois issus de cultures ou d'époques différentes, et comment les éléments d'un texte entrent en écho avec des discours et des facteurs culturels qui influencent la production de ce texte. En bref, le texte doit être compris dans son contexte.

Je n'ai rien à redire à leur critique du formalisme et du structuralisme, mais je crains qu'ils ne fassent tort aux folkloristes et à eux-mêmes, en écartant l'étude des influences orales et du folklore dans leur analyse des contes de Perrault et de ceux écrits par des auteurs d'Ancien Régime. En développant cette notion de "généricité», U. Heidmann souligne l'importance du concept de re-configuration qui montre comment la configuration d'un genre comme le conte de fée subit des transformations.

Ce concept permet de comprendre l'inscription d'énoncés dans des systèmes de genres existants comme une tentative d'infléchir les conventions génériques en vigueur et de créer de nouvelles conventions génériques, mieux adaptées aux contextes socioculturels et discursifs qui changent d'une époque et d'une sphère culturelle et linguistique à l'autre. Par rapport au concept de généricité auquel nos recourons pour désigner plus généralement la dynamique et les fluctuations dont relève toute inscription (auctoriale, éditoriale et lectoriale) d'un énoncé dans un système de genres, le concept de (re)configuration doit permettre de distinguer dans ces fluctuations générales des phases et des contours plus précis ${ }^{14}$.

Tout cela est bien beau mais U. Heidmann et J.-M. Adam écartent toute référence à l'oralité et aux origines probables des pratiques sociales de la narration qui pourraient avoir aidé Perrault à reconfigurer ses textes. Ils minimisent les preuves écrites et picturales qui montrent clairement comment Perrault a pu être influencé par les histoires des nourrices ou des petites gens. Ils écartent l'importance de l'illustration figurant sur la couverture du livre de Perrault : une vieille fille racontant des histoires

I3. U. Heidmann et J.-M. Adam, Textualité et intertextualité des contes: Perrault, Apulée, La Fontaine, Lhéritier, p. I9.

I4. Ibid., p. 34-35. 
à des enfants de bonne famille. De façon plus significative encore, ils ne semblent pas comprendre que certaines de leurs découvertes intertextuelles les plus importantes sont ancrées dans la tradition orale de la Bible et des mythes grecs et romains. Dans son interprétation brillante du Petit Chaperon rouge et de La Barbe bleue de Perrault, U. Heidmann démontre que Perrault s'est fortement inspiré de L'Âne d'or, roman latin écrit par Apulée au $\mathrm{II}^{\mathrm{e}}$ siècle ap. J.-C., et des Contes galants de La Fontaine pour reconfigurer ses contes qui n'étaient pas nommés ainsi à l'époque, mais qui l'ont été depuis. C'est essentiellement sur la base d'une analyse intertextuelle hautement sophistiquée et spécialisée que U. Heidmann et J.-M. Adam définissent leurs interprétations. En privilégiant l'écrit et la littérature, ils établissent une hiérarchie des influences et des sources qui contribuent à la production et à la réception d'une œuvre. Le fait que Perrault reconfigurait également des histoires orales qui faisaient partie de son habitus narratif et qu'il utilisait un style rationnel et viril pour rompre avec la littérature classique et les modes de narration populaires n'est pas pris en considération. U. Heidmann et J.-M. Adam ne considèrent pas non plus les profondes influences orales présentes dans les mythes littéraires qu'ils citent et qui furent disséminés pendant des centaines d'années sur les scènes de théâtre, dans les narrations orales, les salons, les écoles et d'autres lieux publics. Nous ne devons pas oublier que les Grecs et les Romains vivaient leurs mythes. Notons que le conte Cupidon et Psyché, bien que ses origines orales aient été mises en doute, provient des rites oraux des Grecs et des Romains ${ }^{15}$. On peut se référer également au conte de «la femme du charmeur de serpent» dans Bereshit Rabba ${ }^{16}$ et au mythe de Prométhée avec la boîte de Pandore; autant d'histoires qui ont fourni le matériel nécessaire à Perrault pour élaborer sa Barbe bleue. D'ailleurs il est probable que, jusque dans les années I690 à Paris, la plupart des mythes grecs et romains aient circulé aussi bien dans des versions orales que dans des versions imprimées.

Mon but en remettant en cause la notion de "généricité» de U. Heidmann et J.-M. Adam n'est pas de prouver qu'ils ont " tort ${ }^{17}$, mais de me demander pourquoi ils se sont refusés à inclure dans leurs analyses les études historiques traitant du folklore; pourquoi ils n'explorent pas l'habitus narratif de Perrault; et pourquoi ils ont refusé d'établir une distinction

\footnotetext{
I5. Voir le chapitre très intéressant "The Core of a Tale», dans W. Burkert, Creation of the Sacred: Tracks of Biology in Early Religions, p. 56-79.

16. Voir S. Barzilai, "The Snake-Charmer's Wife in Genesis Rabbah», p. I-2I.

I7. N.D.T. : souligné par l'auteur.
} 
parmi les folkloristes, qui, semblent-ils croire, sont tous essentiellement des universalistes. Bien sûr, nous avons une grande dette envers les analyses philologiques et linguistiques de U. Heidmann et J.-M. Adam, qui veulent en fait réhabiliter les contes de Perrault au sein de la littérature, c'est-à-dire au sein de textes littéraires complexes qui reposent sur des références intertextuelles. Mais C. Verevis fait une remarque tout à fait opportune à propos des chercheurs qui, de façon paradoxale, adoptent un point de vue trop étroit quand ils se concentrent presque exclusivement sur l'intertextualité et délaissent l'extratextualité :

Alors qu'une compétence générale et cinématographique permet l'élaboration d'une hiérarchie des intrigues déterminée par l'intertexte — de la plus succincte à la plus détaillée - la construction d'une relation intertextuelle spécifique entre un remake et son original présumé est un acte d'interprétation ${ }^{18}$; un acte qui est «limité et relatif — non pour un sujet [spectateur] mais pour la grille d'interprétation (le régime de lecture) à travers laquelle la position du sujet et les relations textuelles sont constituées». Enfin, et comme Frow lui-même le soutient en ce qui concerne ce concept d'intertextualité, ce qui fait la valeur du remake ce n'est pas «l'identification en détail [...] de source $[s]$ intertextuelles spécifiques ", qui servent rétrospectivement de points d'origine, mais la détermination "d'une structure discursive plus générale : le genre du "remake" destiné à la re-visualisation".

Bien que leur théorie de généricité et de reconfiguration prétende être interdisciplinaire et embrasser les circonstances socioculturelles, U. Heidmann et J.-M. Adam étudient l'évolution du conte en tant que genre à travers des mouvements littéraires ${ }^{19}$ d'intertextualité et d'interculturalité qui jaillissent et rejaillissent, qui configurent et reconfigurent pour apporter de l'innovation littéraire. De ce point de vue, la théorie de C. Verevis sur le remake vient compléter leur travail et elle peut nous permettre d'apprécier la signification du film de Breillat par rapport à la tradition discursive de La Barbe bleue, et de voir comment les circonstances socioculturelles à travers lesquelles Breillat a repensé La Barbe bleue se reflètent dans la tradition culturelle du conte et de la narration qui a amené Perrault à écrire son texte. En effet, sa conception complète du remake, qui inclut les notions de généricité et de mémétique sans toutefois s'y référer explicitement, est capitale pour comprendre pourquoi le film de Breillat a attiré autant l'attention, pourquoi il peut marquer un tournant dans la façon d'appréhender le conte de Perrault aujourd'hui, et pourquoi

I8. N.D.T. : souligné par l'auteur.

I9. N.D.T. : souligné par l'auteur. 
ce film contribue à la mise en scène du conte et à un discours culturel plus vaste sur les tueurs en série et la curiosité féminine.

\section{Le remake de La Barbe bleue selon Breillat}

Tout comme les femmes auteurs de contes faisaient partie d'un mouvement protoféministe dans les années I690 (et Perrault fut influencé par ces femmes de lettres), Breillat s'en inspira et joua un rôle significatif dans la seconde vague de féminisme contemporain en France. Et donc, sa reconfiguration de La Barbe bleue de Perrault doit être située dans le mouvement féministe français qui débuta à la fin des années I690, et qui continua par la suite à modifier les habitudes culturelles et sociales en France. Née en I948, Breillat fut une féministe avant la lettre ${ }^{20}$. Venant d'une petite ville de l'ouest de la France, elle s'installa à Paris à l'âge de seize ans avec l'espoir de devenir réalisatrice. Cependant elle ne fut pas admise à l'Institut des Hautes Études Cinématographiques et dut se contenter de petits rôles au cinéma. En parallèle, elle commença à écrire des histoires, des poèmes et des essais, et en 1968 elle publia son premier roman L'Homme facile (traduit en anglais sous le titre $A$ Man for the Asking), qui connut un succès immédiat grâce à ses scènes de sexe cru. Que ce soit avec ses textes, ses rôles ou ses mises en scène, Breillat a gagné une réputation de provocatrice $^{21}$ qui explorait la sexualité des femmes et la honte qu'elles ont été forcées de ressentir sous le regard des hommes. Presque tous ses plus grands romans et films se font les portraits nullement timides des relations sexuelles magnifiquement décrites comme d'intenses enchevêtrements et luttes des corps, dans lesquels les femmes cherchent à se comprendre ellesmêmes en découvrant et en revendiquant leurs désirs sexuels. De plus, certains de ses films comme $\grave{A}$ ma saur (également connu sous le titre anglais Fat Girl, sorti en 200I) traitent de la rivalité entre frères et sœurs, ou de la compétition entre filles ou femmes qui implique une expression sexuelle libérée.

Vraisemblablement, Breillat ne s'intéressa pas beaucoup à la transformation féministe et radicale du conte pendant la dernière partie du $\mathrm{xx}^{\mathrm{e}}$ siècle, même si la plupart des contes anglo-américains les plus importants écrits par Margaret Atwood, Angela Carter, A. S. Byatt, et bien d'autres encore étaient connus en France. Par ailleurs, il y eut en France plusieurs femmes

20. N.D.T. : en français dans le texte et souligné par l'auteur.

2I. N.D.T. : en français dans le texte et souligné par l'auteur. 
écrivains célèbres, comme Pierrette Fleutiaux qui a mis sens dessus dessous les contes de Perrault dans son remarquable recueil, Métamorphoses de la reine, publié en 1984. Mais Breillat était davantage influencée par les œuvres de Marguerite Duras et Hélène Cixous. Et comme Douglas Keesey l'a précisé,

Breillat peut être considérée comme faisant partie d'un groupe de femmes écrivains de plus en plus nombreuses qui sont pour la plupart non-féminines dans leurs confrontations sexuelles directes mettant en jeu certains aspects particulièrement sombres de la passion. Parmi ces auteurs, citons Virginie Despentes (avec Baise-moi, publié en 1994), Marie Darrieussecq (Truismes, en 1996), Catherine Cusset (Jouir, en 1997), Christine Angot (L'Inceste, en 1999), Camille Laurens (Dans ces bras-là, en 2000) et Catherine Millet (La Vie sexuelle de Catherine M., en 200I) ${ }^{22}$.

Les œuvres de ces auteurs, les lectures de sa jeunesse, les films qu'elle a visionnés et produits, et les nombreuses explications des contes et de ses propres histoires formèrent l'habitus narratif de Breillat.

Malgré le fait que Breillat n'ait montré aucun intérêt artistique pour les contes de fées jusqu'en 2005, quand elle commença à travailler sur l'adaptation de La Barbe bleue de Perrault, elle déclara dans plusieurs interviews que ce conte avait toujours été l'un de ses préférés à elle et à sa sœur aînée, et que l'idée d'une adaptation filmée de La Barbe bleue lui trottait dans la tête depuis au moins douze ans avant qu'elle ne se lançât dans la réalisation de son projet sous la forme d'un film ${ }^{23}$. Il est utile de rappeler ici que Breillat est la première ${ }^{24}$ femme metteur en scène à avoir adapté un conte à l'écran — celui-ci le fut tout d'abord pour la télévision —, non seulement en France mais aussi dans toute l'Europe. Depuis 1945 et tous pays confondus, il y a eu environ vingt films tirés de La Barbe bleue de Perrault; les versions françaises les plus importantes ont été réalisées par

\footnotetext{
22. D. Keesey, Catherine Breillat, p. I5I.

23. Par exemple, nous pouvons nous reporter à son interview avec N. Poinsot, «La scandaleuse, Catherine Breillat lance Barbe-Bleue au festival du film fantastique de Neuchâtel», dans laquelle elle déclare: "Comme beaucoup de contes, celui-ci aborde un thème essentiel. Pour ma sœur et moi, il a toujours été notre préféré, depuis l'enfance. On le lisait à haute voix et on se mettait à pleurer ensemble avant d'arriver à la fin. D'une certaine façon, il s'adresse surtout aux petites filles. Il leur montre comment apprendre à aimer l'homme qui va les tuer. Leurs illusions, elles les perdent très vite, parce qu'à quatorze ans, beaucoup se trouvent déjà vieilles. [...] En fait ce film mûrissait dans ma tête depuis une dizaine d'années, mais il a véritablement commencé à prendre forme en 2005.» (En ligne: http://www.lesquotidiennes.com/culture/la-scandaleuse-catherinebreillat-lance-barbe-bleue)

24. N.D.T. : souligné par l'auteur.
} 
Christian-Jaque, Claude Chabrol, Alexandre Bubnov, et Pierre Boutron ${ }^{25}$. Mais aucun des réalisateurs de ces vingt films, dont Charlie Chaplin et son extraordinaire Monsieur Verdoux de I947, n'a adapté le conte avec une photographie aussi délicate et avec une focalisation aussi précise sur le désir passionné de s'affirmer d'une jeune femme que Breillat. Dans une certaine mesure, c'est parce que Breillat vit et respire à travers toutes ses histoires et ses tournages qu'elle a réussi à transformer La Barbe bleue de Perrault en un film qui explore la rébellion d'une jeune femme plutôt que sa victimisation. Breillat a conçu l'idée du film, a écrit le scénario, a trouvé le lieu du tournage dans le nord de la France, a auditionné les acteurs, dont certains étaient des amateurs, a créé certains décors et costumes, et a mis au point la version finale. Des références autobiographiques directes à la rivalité qui existait entre elle et sa sœur marquent le film. Le monstrueux tueur en série Barbe bleue est dépeint ironiquement comme un intellectuel solitaire. Il revêt presque une importance secondaire parce que les deux jeunes protagonistes, Catherine, la narratrice âgée de six ans, et Marie-Catherine, la jeune femme qui épouse Barbe bleue, dominent l'action dans la mesure où elles prennent en charge les récits de leur propre vie.

Au début, le spectateur ne pense pas que le film traitera d'une narratrice contestant le récit du mâle dominant, ou du regard de la femme contre le regard de l'homme, ou de Breillat contre Perrault. Mais il traite en fait de tous ces conflits et même d'autres encore. Contrairement au conte de Perrault, le film de Breillat débute sur une note triste. La scène d'ouverture se déroule quelque part dans le nord de la France dans un couvent froid et aux allures de prison. Deux sœurs, Marie-Catherine (qui a environ dixsept ans) et Anne (environ dix-neuf ans), sont convoquées par l'impitoyable mère supérieure qui les informe que leur père vient de mourir dans un accident de la route. Elle les expulse littéralement de l'école catholique privée parce qu'elles ne peuvent en payer les frais. Une fois que nous, le public, sommes confrontés au sort de ces deux filles du XviI ${ }^{\mathrm{e}}$ siècle, Breillat nous fait entrer sans transition dans une vieille ferme pendant les années I950 en France : dans la grange, on voit deux filles qui montent au grenier et qui commencent à explorer les merveilleux objets entreposés là. Â partir de ce moment, Breillat passe d'une époque à l'autre : elle filme des histoires parallèles appartenant au passé, les années I690 et 1950, pour réfléchir sur les problèmes rencontrés par les jeunes filles d'aujourd'hui car

25. Voir le chapitre «Bluebeard's Original Sin and the Rise of Serial Killing, Mass Murder, and Fascism» dans mon ouvrage The Enchanted Screen: The Unknown History of the Fairy-Tale Film, p. 158-17I. 
il y a toujours des tueurs en série. Et il y a toujours de la rivalité entre frères et sœurs. Certains critiques ont vu dans la construction du film de Breillat des histoires enchâssées, mais ce n'est pas le cas. Breillat fait se refléter deux récits distincts mais similaires et use du redoublement pour créer un effet d'éloignement, et pour inciter le public à comparer et à mettre en contraste les deux histoires. Il n'y a là aucune trame de conte, à moins de considérer que Breillat filme les histoires au sein même de la trame d'un récit de La Barbe bleue dont les origines profondes se trouvent dans une tradition orale et littéraire en France et ailleurs. Cette tradition orale et littéraire fournit de façon mémétique sa trame au film.

Les deux intrigues sont bien distinctes. En ce qui concerne les sœurs du XVII ${ }^{\mathrm{e}}$ siècle, la plus jeune des deux, Marie-Catherine, jure de se venger de la cruelle mère supérieure car elle a le projet de faire un beau mariage, de devenir riche et de vivre dans un château semblable à celui habité par Barbe bleue. Elle refuse d'accepter sa condition de jeune fille pauvre. Bien qu'elle entende dire que Barbe bleue est un dangereux personnage, elle gagne vite sa confiance, l'épouse et prend l'ascendant sur lui. Un gentil géant que cet homme lorsqu'il remarque que Marie-Catherine est innocente comme une blanche colombe et fière comme un coq. C'est le coq qui est en elle qui finalement fera tomber la tête du méchant.

En ce qui concerne les sœurs du Xx ${ }^{\mathrm{e}}$ siècle, Catherine, la petite fille déterminée, trouve un recueil de contes de Perrault dans le grenier et commence la lecture de La Barbe bleue à sa grande sœur, plus sensible et d'ailleurs effrayée par l'histoire. L'histoire lue par Catherine n'est pas une copie fidèle du conte de Perrault, et les différentes scènes qu' elle imagine ne le sont pas non plus. Ce que l'on peut retenir de sa lecture, c'est la manière avec laquelle elle s'approprie le conte et fait de cette histoire et de ces aventures les siennes. Elle s'identifie clairement à cette Marie-Catherine dans l'histoire qu'elle s'invente. Catherine prend également un plaisir sadique à effrayer sa grande sœur et cause accidentellement sa mort : tout en poursuivant sa lecture, elle fait reculer sa sœur qui tombe à travers une trappe du grenier.

Le redoublement des personnages tout au long du film permet à Breillat de modifier la perspective du récit à la troisième personne de Perrault et de le remettre en question. De même, les deux jeunes "protagonistes" prennent le contrôle sur les récits de leur vie, pour le meilleur ou pour le pire, de même, Breillat radicalise le conte de Perrault pour exposer l'oppression des jeunes femmes et ses propres expériences avec sa sœur. L'œil de la caméra explore des aspects du récit de Perrault selon des points de vue inhabituels qui se concentrent davantage sur les choix des femmes que sur le mystère qui se cache derrière les meurtres de Barbe bleue. Breillat 
rapproche les histoires mises en miroir seulement une fois, lorsque l'on voit la petite Catherine entrer dans la pièce tachée de sang où trois des femmes tuées par Barbe bleue sont suspendues le long d'un mur. Mise à part cette scène, la version filmée de Breillat ne rapproche pas les deux histoires et les deux paires de sœurs sont très différentes.

L'une des deux intrigues illustre le désir profond d'une jeune fille qui veut échapper à la pauvreté et vivre dans un monde de conte de fées. Au fur et à mesure elle apprend à connaître Barbe bleue, partage sa solitude et, après qu'il ait été tué, caresse de façon étrange sa tête décapitée. Le monde du conte de fées n'est pas aussi joyeux et onirique que les jeunes filles le souhaiteraient. La seconde histoire parle plutôt de la rivalité entre sœurs : une petite fille pleine d'assurance déforme le conte de Perrault de telle sorte qu'elle prend l'ascendant sur sa sœur aînée et la tue. Contrairement à $L a$ Barbe bleue de Perrault, les deux histoires de Breillat ont une fin tragique et laissent pourtant la porte ouverte à d'autres histoires. Marie-Catherine est représentée, comme dans un tableau, avec la tête de Barbe bleue posée sur un plateau, et nous n'avons aucune idée de ce qu' elle va faire ensuite. Il en va de même pour la petite Catherine qui se trouve aux côtés de sa mère alors qu'elles regardent le corps de sa sœur, morte suite à sa chute au travers de la trappe. Mais nous savons avec certitude que le remake de Breillat a révolutionné la tradition mémétique des contes autour du personnage de Barbe bleue, en adoptant de nouveaux points de vue en phase avec le rôle des femmes à la fin du $\mathrm{Xx}^{\mathrm{e}}$ et au début du XXI ${ }^{\mathrm{e}}$ siècle.

\section{Réflexions sur les tueurs en série, le rôle des femmes et les récits de La Barbe bleue}

Il est pratiquement impossible de dater le début d'une réflexion orale et littéraire sur les tueurs en série et la curiosité féminine. Dans son remarquable ouvrage, Fairytale in the Ancient World, Graham Anderson écrit :

L'association d'un bon nombre de schémas hybrides est souvent nécessaire avant de pouvoir comprendre que la plupart des caractéristiques dès lors connues dans un conte existaient déjà dans l'Antiquité à travers certains rapports équilibrés qu'elles entretenaient les unes aux autres. Nous sommes également rarement capables d'établir une quelconque progression débouchant sur la construction de l'archétype d'un conte donné. Les différentes versions que l'on peut trouver dans la littérature héritée de l'Antiquité sont au moins aussi variées et obscures que leurs équivalents d'aujourd'hui, suggérant que les contes eux-mêmes sont déjà vieux ${ }^{26}$.

26. G. Anderson, art. cité, p. IO2. 
Dans le cas de La Barbe bleue, j'ai déjà mentionné qu'il y a de "vieux ${ }^{27}$ » motifs et éléments que Perrault a insérés dans son texte: les histoires bibliques comme celle d' "Adam et Eve", le midrash qui commente le Livre de la Genèse, et les histoires de l'Antiquité comme celles de la "boîte de Pandore» et du «Roi Minos de Crète». On pourrait même ajouter la trame du conte des Mille et Une Nuits, précurseur du conte hybride La Barbe bleue. Après tout, le roi Shahryar, qui épouse des jeunes filles vierges le jour et les tue après avoir passé la nuit avec elles, est le plus célèbre des tueurs en série de toute l'humanité.

Dans l'évolution des histoires qui contribuèrent à la formation du genre littéraire du conte, et en particulier de récits autour de Barbe bleue, on voit que ces histoires avaient une vie qui leur était propre et qu'elles étaient véhiculées par les hommes et les relations sociales qu'ils avaient entre eux. Et en effet, c'est toujours vrai aujourd'hui. Dans sa brillante analyse, Letting Stories Breathe: A Socio-Narratology, Arthur Frank insiste sur le fait que «les histoires fonctionnent avec, pour, et toujours sur ${ }^{28}$ les gens, touchant ce qu'ils peuvent considérer comme étant aussi vrai que possible, comme étant à faire ou à ne pas faire. [...] Les histoires insufflent la vie non seulement aux individus, mais aussi aux groupes qui se réunissent pour raconter ou pour croire en certaines histoires ${ }^{29} »$.

Il n'est pas inconcevable d'imaginer que, des milliers d'années en arrière, les gens racontèrent de nombreuses histoires sur des tueurs en série afin de donner l'alerte sur ces meurtriers. On peut concevoir que les gens racontèrent également des histoires sur la curiosité des femmes et le fait qu'elles pouvaient se mettre en danger si elles étaient trop curieuses et désobéissaient à un mari despotique. Comme Shuli Barzilai l'a remarqué, l'intrigue des contes qui contribuèrent au développement des récits autour de Barbe bleue comprend les phases d'interdiction, de transgression et de punition $^{30}$. Ces trois phases apparaissent dans de nombreux types de contes, mais ce qui est frappant avec les récits concernant Barbe bleue c'est qu'ils prennent leur source dans une façon de raconter empreinte de tendances misogynes provenant de sociétés patriarcales. La Barbe bleue est un conte sur le pouvoir, entre autres choses, et sur celui qui détient le pouvoir, et sur

27. N.D.T. : souligné par l'auteur. 28. N.D.T. : souligné par l'auteur.

29. A. Frank, ouvr. cité, p. 3.

3o. S. Barzilai, art. cité, p. 5 . 
la raison pour laquelle le pouvoir devrait toujours rester dans les mains de l'homme ${ }^{31}$. Comme S. Barzilai l'indique :

Si la peur de la sexualité débridée des femmes dépend du contexte sociopolitique, comme Pandore le suggère, métaphoriquement, en brisant le sceau d'une boîte et en en soulevant le couvercle, ce sont en effet les thèmes du pouvoir et de la domination qui produisent les histoires successives destinées à représenter l'humiliation des femmes et à justifier l'hégémonie des hommes. L'affirmation de la domination de l'homme dépend dialectiquement de la faillibilité de la femme. Selon des termes empruntés à Jacques Lacan, le mari est constamment obligé de chercher à se rassurer quant à l'emplacement de son phallus ${ }^{32}$.

La signification et la popularité de La Barbe bleue de Perrault peuvent être attribuées à cet assemblage subtil et artistique de divers motifs et thèmes qui mettent l'accent sur le pouvoir masculin et sur la curiosité féminine, ainsi qu'à son invention du personnage emblématique de Barbe bleue. Perrault se nourrissait de son conte et le respirait. Il lui donna vie grâce à son immense talent de narrateur et d'écrivain de sorte que ce conte devint mémorable et mémétique. De plus, à travers sa réécriture ${ }^{33}$ d'autres histoires qu'il rassembla dans ce conte, il modela avec brio son propre récit de telle manière qu'il permit la remise en cause de l'hégémonie de l'homme, et provoqua d'autres réflexions à travers ses deux moralités ${ }^{34}$ concernant la curiosité des femmes et les relations changeantes entre les hommes et les femmes. Ironiquement, il inventa le nom d'un conte-type discursif qui devait éliminer son propre nom, car La Barbe bleue, conte qui vit toujours, est devenu plus important que le nom ou l'existence de l'auteur lui-même.

Breillat a donné une autre vie au récit de Barbe bleue en recréant le conte de Perrault à travers l'écriture du scénario et la mise en scène de sa Barbe bleue. Elle en a fait une refonte radicale parce qu'elle a complexifié l'intrigue de l'interdiction, de la transgression et de la punition, et a mis l'accent sur l'affirmation du pouvoir des femmes et non sur leur curiosité. Il n'y a pas un soupçon de misogynie dans son film; elle n'épanche pas non plus sa bile contre l'oppression de la femme en décrivant Barbe bleue comme un barbare. Comme je l'ai déjà mentionné, il ressemble plutôt à un intellectuel solitaire qu'à un monstre. À la fin du film, Marie-Catherine caresse amoureusement la tête de Barbe bleue comme si elle avait pitié de

\footnotetext{
3I. On peut se référer à ma réflexion sur ce thème dans "The Male Key to Bluebeard's Secret", Why Fairy Tales Stick: The Evolution and Relevance of a Genre, p. I55-194. Voir aussi P. Lewis, Seeing Through the Mother Goose Tales: Visual Turns in the Writings of Charles Perrault.

32. S. Barzilai, art. cité, p. 8.

33. N.D.T. : souligné par l'auteur.

34. N.D.T. : souligné par l'auteur.
} 
lui. Le temps de la domination arbitraire de l'homme semble prendre fin. Mais comme Breillat le sait elle-même, ce n'est pas le cas. Son histoire marque certainement une rupture dans les récits sur Barbe bleue car elle fait partie d'un grand mouvement féministe culturel conditionnant la narration et le remake de ce conte, mouvement qui débuta dans les années I970 et perdurera au XXI ${ }^{\mathrm{e}}$ siècle. Aussi longtemps qu'il y aura des tueurs en série et que le patriarcat exigera l'asservissement des femmes, nous aurons de nouvelles versions de La Barbe bleue qui représenteront des conflits de société et des solutions envisageables. Le film de Breillat montre qu'il est peut-être temps de dire au-revoir aux propositions de Perrault, mais il est indéniable qu'elle n'aura pas le dernier mot.

Traduction de l'anglais : Cécile Boulic

\section{Bibliographie}

BArzilaI Shuli, "The Snake-Charmer's Wife in Genesis Rabbah, or Bluebeard Begins", dans Tales of Bluebeard and his Wives from Late Antiquity to PostmodernTimes, Londres, Routledge, 2009.

Burkert Walter, Creation of the Sacred: Tracks of Biology in Early Religions, Cambridge, Harvard University Press, 1996.

Davies Mererid Puew, The Tale of Bluebeard in German Literature: From the Eighteenth Century to the Present, New York, Oxford University Press, 200I.

Distin Kate, The Selfish Meme, Cambridge, Cambridge University Press, 2005.

Drout Michael, "A Meme-Based Approach to Oral Traditional Theory», Oral Tradition, vol. 2I, $\mathrm{n}^{\circ}$ 2, 2006.

Heidmann Ute et Adam Jean-Michel, Textualité et intertextualité des contes: Perrault, Apulée, La Fontaine, Lhéritier, Paris, Garnier, 2010.

Hermansson Casie, Bluebeard: A Reader's Guide to the English Tradition, Jackson (Michigan), University Press of Mississippi, 2009.

Keesey Douglas, Catherine Breillat, Manchester, Manchester University Press, 2009.

Lewis Philip, Seeing Through the Mother Goose Tales: Visual Turns in the Writings of Charles Perrault, Stanford (Californie), Stanford University Press, 1996.

Perrault Charles, Contes de ma Mère l'Oye, H. Tronc (éd.), Paris, Gallimard, 2006. 
Un remake de La Barbe bleue, ou l'au-Revoir à Perrault

Poinsoт Nicolas, «La scandaleuse, Catherine Breillat lance Barbe-Bleue au festival du film fantastique de Neuchâtel ", http://www.lesquotidiennes. $\mathrm{com} /$ culture/la-scandaleuse-catherine-breillat-lance-barbe-bleue

Tatar Maria, Secrets beyond the Door: The Story of Bluebeard and his Wives, Princeton (New Jersey), Princeton University Press, 2004.

Verevis Constantine, Film Remakes, Édimbourg, Edinburgh University Press, 2006.

Zipes Jack, Why Fairy Tales Stick: The Evolution and Relevance of a Genre, New York, Routledge, 2006.

-, The Enchanted Screen: The Unknown History of the Fairy-Tale Film, New York, Routledge, 20II. 